\title{
Performing clinical 18F-FDG-PET/MRI of the mediastinum optimising a dedicated, patient-friendly protocol
}

\author{
Citation for published version (APA):
}

Peerlings, J., Paulis, L., Mitea, C., Bakers, F., Berbee, M., Wierts, R., Voo, S., Wildberger, J., Hoffmann, A., Lambin, P., \& Mottaghy, F. (2019). Performing clinical 18F-FDG-PET/MRI of the mediastinum optimising a dedicated, patient-friendly protocol. Nuclear Medicine Communications, 40(8), 815-826. https://doi.org/10.1097/MNM.0000000000001035

Document status and date:

Published: 01/08/2019

DOI:

10.1097/MNM.0000000000001035

Document Version:

Publisher's PDF, also known as Version of record

\section{Document license:}

Taverne

Please check the document version of this publication:

- A submitted manuscript is the version of the article upon submission and before peer-review. There can be important differences between the submitted version and the official published version of record.

People interested in the research are advised to contact the author for the final version of the publication, or visit the DOI to the publisher's website.

- The final author version and the galley proof are versions of the publication after peer review.

- The final published version features the final layout of the paper including the volume, issue and page numbers.

Link to publication

\footnotetext{
General rights rights.

- You may freely distribute the URL identifying the publication in the public portal. please follow below link for the End User Agreement:

www.umlib.nl/taverne-license

Take down policy

If you believe that this document breaches copyright please contact us at:

repository@maastrichtuniversity.nl

providing details and we will investigate your claim.
}

Copyright and moral rights for the publications made accessible in the public portal are retained by the authors and/or other copyright owners and it is a condition of accessing publications that users recognise and abide by the legal requirements associated with these

- Users may download and print one copy of any publication from the public portal for the purpose of private study or research.

- You may not further distribute the material or use it for any profit-making activity or commercial gain

If the publication is distributed under the terms of Article 25fa of the Dutch Copyright Act, indicated by the "Taverne" license above, 


\section{Performing clinical ${ }^{18}$ F-FDG-PET/MRI of the mediastinum optimising a dedicated, patient-friendly protocol} Jurgen Peerlings s, ${ }^{a, b, c, d}$, Leonie Paulis ${ }^{a}$, Cristina Mitea ${ }^{a, d}$, Frans Bakers ${ }^{a}$, Maaike Berbée ${ }^{b}$, Roel Wierts ${ }^{a}$, Stefan Vöö ${ }^{a, e}$, Joachim Wildberger ${ }^{a}$, Aswin Hoffmann ${ }^{b, f, g, h}$, Philippe Lambin ${ }^{c, d}$ and Felix Mottaghy ${ }^{a, i}$

Objective To construct a mediastinal-specific fluorine18-fluorodeoxyglucose $\left({ }^{18} \mathrm{~F}\right.$-FDG)-PET/MR protocol with high-quality MRI of minimal acquisition-time and comparable diagnostic value to ${ }^{18} \mathrm{~F}-\mathrm{FDG}$-PET/computed tomography (CT).

Materials and methods Fifteen healthy participants received PET/MRI and 10 patients with mediastinal tumours (eight non-small-cell lung, two oesophageal cancer) received ${ }^{18} \mathrm{~F}$-FDG-PET/MRI immediately after ${ }^{18} \mathrm{~F}$-FDG-PET/CT. Sequences volume interpolated breathhold examination (T1-VIBE) and Half-Fourier acquisition single-shot turbo spin echo (T2-HASTE) were optimised by varying the parameters: breath-hold $(\mathrm{BH}$, endexpiration), fat suppression (spectral adiabatic inversion recovery), and ECG-triggering (ECG, end-diastole). Image quality (IQ) of each sequence-variation was qualitatively scored by medical experts and quantitatively assessed by calculating signal-to-noise ratios, contrast relative to muscle, standardized-uptake-value, and tumour-toblood ratios. Patient comfort was evaluated on patients' experience. Diagnostic accuracy of ${ }^{18} \mathrm{~F}$-FDG-PET/ MRI was compared to ${ }^{18} \mathrm{~F}-\mathrm{FDG}-\mathrm{PET} / \mathrm{CT}$, in reference to histopathology/cytopathology.

Results ECG-triggered T1-VIBE images showed the highest signal-to-noise ratio $(P<0.01)$ and the largest contrast between mediastinal soft-tissues, regardless of $\mathrm{BH}$ or free-breathing acquisition. IQ of ECG-triggered T1-VIBE scans in BH were scored qualitatively highest with good reader agreement $(\kappa=0.62)$. IQ of T2-HASTE was not significantly affected by $\mathrm{BH}$ acquisition $(P>0.9)$. Qualitative IQ of T1-VIBE and T2-HASTE declined after

\section{Introduction}

Hybrid imaging, combining PET with either computed tomography (i.e. PET/CT) or MRI (i.e. PET/MRI), has been implemented in a variety of oncological management steps [1,2]. In thoracic oncological imaging, PET/ CT is considered to be the current clinical standard. Nevertheless, an additional MR scan is often needed

Supplemental Digital Content is available for this article. Direct URL citations appear in the printed text and are provided in the HTML and PDF versions of this article on the journal's website, www.nuclearmedicinecomm.com. spectral adiabatic inversion recovery fat-suppression. All patients could maintain $\mathrm{BH}$ at end-expiration and reported no discomfort. Diagnostic performance of ${ }^{18} \mathrm{~F}$-FDG-PET/ $M R$ was not significantly different from ${ }^{18} \mathrm{~F}$-FDG-PET/CT with comparable staging, standardized-uptake-values, and tumour-to-blood ratios. However, T-status was more often over-staged on ${ }^{18} \mathrm{~F}$-FDG-PET/CT, while $\mathrm{N}$-status was more frequently under-staged on ${ }^{18} \mathrm{~F}$-FDG-PET/MR.

\section{Conclusion ECG-triggered T1-VIBE sequences} acquired during short, multiple $\mathrm{BHs}$ are recommended for mediastinal imaging using ${ }^{18} \mathrm{~F}-\mathrm{FDG}$-PET/MR. With dedicated protocols, ${ }^{18} \mathrm{~F}$-FDG-PET/MRI will be useful in thoracic oncology and aid in diagnostic evaluation and tailored treatment decision-making. Nucl Med Commun 40:815-826 Copyright (c) 2019 Wolters Kluwer Health, Inc. All rights reserved.

Nuclear Medicine Communications 2019, 40:815-826

Keywords: hybrid imaging, mediastinum, non-small-cell lung cancer, oesophageal cancer, PET-CT, PET-MRI

${ }^{a}$ Department of Radiology and Nuclear Medicine, ${ }^{b}$ Department of Radiation Oncology (MAASTRO clinic), 'The D-Lab, Maastricht Comprehensive Cancer Centre, dGROW - School for Oncology and Developmental Biology, Maastricht University Medical Centre, Maastricht, The Netherlands, 'Institute of Nuclear Medicine, University College London Hospital, London, UK, Institute of Radiooncology - OncoRay, Helmholtz-Zentrum Dresden-Rossendorf, ${ }^{9}$ OncoRay - National Centre for Radiation Research in Oncology, hepartment of Radiotherapy and Radiation Oncology, Faculty of Medicine and University Hospital Carl Gustav Carus, Technical University Dresden, Dresden and 'Department of Nuclear Medicine, University Hospital RWTH Aachen University, Aachen, Germany

Correspondence to Jurgen Peerlings, PhD, MSc, Department of Radiology and Nuclear Medicine, Maastricht University Medical Centre, P. Debyelaan 25, 6202 AZ Maastricht, The Netherlands

Tel: +31 088445 5587/+31241808 8740; e-mail: peerlings.j@gmail.com

Received 6 November 2018 Revised 22 April 2019 Accepted 24 April 2019

to clarify equivocal findings, detect tumour infiltration, and differentiate metastatic lymph nodes (LNs) [3-5]. It was generally anticipated that with the development of integrated PET/MR systems, the diagnostic accuracy would improve for these situations. However, a clear clinical improvement of whole-body fluorine-18-fluorodeoxyglucose $\left({ }^{18} \mathrm{~F}\right.$-FDG)-PET/MR over ${ }^{18} \mathrm{~F}$-FDGPET/CT has yet to be determined in oncology [6]. An alternative approach using region-specific instead of whole-body ${ }^{18} \mathrm{~F}$-FDG-PET/MRI-protocols could lead to more insight into the efficient application and further 
integration of PET/MR in thoracic oncological imaging. Here, PET-images could remain the leading diagnostic tool by detecting ${ }^{18} \mathrm{~F}$-FDG-avid lesions with high sensitivity, while MRI of ${ }^{18} \mathrm{~F}$-FDG-positive regions would be better suited as the matching tool than CT to determine the underlying pathological correlate (e.g. tumour infiltration, mediastinal $\mathrm{LN}$ involvement, inflammation).

For new imaging modalities and protocols to be adopted an efficient patient workflow must be developed. Hybrid imaging protocol development rests on three fundamental pillars: (a) producing clinically valuable images with high image quality (IQ) and diagnostic accuracy, (b) implementing time-efficient protocols by simultaneous acquisition of PET and MRI, (c) efficient acquisition of complementary, nonredundant data [7]. For successful mediastinal-specific protocols, also image-distorting MR-artefacts related to respiratory and cardiac motion need to be minimised using, for example, cardiac triggering and breath-hold $(\mathrm{BH})$ techniques, while preserving patient comfort. However, patients with non-small-cell lung cancer (NSCLC) often present dyspnoea and may thus find it problematic to maintain a $\mathrm{BH}$ for a certain period of time. Therefore, MRI sequences have to be tailored to this specific patient population.

To this end, the goal of this study was to obtain a mediastinal-specific clinical ${ }^{18} \mathrm{~F}$-FDG-PET/MR protocol containing dedicated MRI-sequences able to produce high-quality images with optimal patient compliance and diagnostic performance comparable to ${ }^{18} \mathrm{~F}$-FDG-PET/ CT. The different T1-weighted/T2-weighted sequences will not be compared with each other. Functional MRI sequences, such as diffusion-weighted (DW) MRI, will not be assessed in this study but might benefit future clinical protocols.

\section{Materials and methods Study population}

Between June 2016 and February 2017, 25 participants were included for PET/MRI after approval by the local Ethics Review Committee (METC 16-4-036). Fifteen adults, healthy volunteers were included to optimise clinical MRI pulse-sequences for imaging mediastinal structures without acquiring PET-images. Next, 10 patients suspected of having mediastinal malignancies (NSCLC $n=8$, oesophageal cancer; EC $n=2$ ) were included to validate the optimised protocol. All patients received a conventional ${ }^{18} \mathrm{~F}-\mathrm{FDG}$ PET/CT scan for primary staging, immediately followed by a dedicated ${ }^{18}$ F-FDG-PET/MRI scan.

\section{Image acquisition}

\section{${ }^{18}$ F-FDG-PET/CT imaging}

Whole-body PET images were obtained on a Gemini TF 64 scanner (Philips Healthcare, Best, NL and Labrador, USA) using the default manufacturer's time-of-flight reconstruction algorithm (BLOB-OS-TF). The injected dose of ${ }^{18} \mathrm{~F}-\mathrm{FDG}$ was $2 \mathrm{MBq} / \mathrm{kg}(162 \pm 34 \mathrm{MBq})$ and administered after patients fasted for $4 \mathrm{~h}$. CT images of $4 \mathrm{~mm}$ thickness were acquired at $120 \mathrm{kV}$ and $30 \mathrm{mAs}$.

\section{${ }^{18}$ F-FDG-PET/MRI}

Mediastinal PET/MRI was performed on a 3-Tesla Magnetom Biograph mMR scanner (Siemens Healthineers, Erlangen, Germany) using two body coils. ${ }^{18} \mathrm{~F}$-FDG-PET data was acquired in list-mode for $15 \mathrm{~min}$ at one bed-position. PET-images were reconstructed using a 3D-iterative ordinary Poisson ordered-subsets expectation maximisation (OP-OSEM3D) reconstruction algorithm (3 iterations, 21 subsets, $4 \mathrm{~mm}$ Gaussian smoothing) and fused with MRI using Syngo.via software (Siemens Healthcare). The dedicated PET/MR-protocol was centred over the carina and included a standard localizer sequence, followed by a 3D low-resolution T1-Dixon-VIBE sequence for MR-based attenuation correction. The IQ and diagnostic value of T1-DixonVIBE sequence have not been assessment in this study [21]. Based on literature and preliminary evaluation of clinically available sequences, two sequences were selected for further optimisation for tumour imaging: (a) T1-weighted Volumetric Interpolated 3D Gradient Echo (T1-VIBE) and (b) T2-weighted Half Fourier Acquisition Single Shot Turbo Spin Echo (T2-HASTE) (Table 1) [8-10]. Sequences were optimised to acquire transversal images of high spatial resolution (in-plane resolution $<1.6 \mathrm{~mm}$ for $\mathrm{LN}$ detection) within a reasonable acquisition time [11].

\section{Variations of mediastinal-specific MR scanning}

Images were acquired using all combinations of MR settings: (a) free-breathing ( $\mathrm{FB}$ ) or $\mathrm{BH}$ acquisition, (b) with or without ECG-triggering, (c) with or without fat suppression.

$\mathrm{BH}$ scans were performed in multiple sessions of four times $5.7 \mathrm{~s}$ for T1-VIBE and seven times $8.1 \mathrm{~s}$ for T2-HASTE.

Table 1 Details of PET/MRI sequences

\begin{tabular}{lcc}
\hline Parameters & T1-VIBE & T2-HASTE \\
\hline Sequence type & Gradient-echo & Spin-echo \\
Acquisition & 3D & 2D \\
Image plane & Transversal & Transversal \\
TR (ms) & 3.64 & 1500 \\
TE (ms) & 1.23 & 113 \\
Flip angle (deg.) & 9 & 90 \\
Number of slices & 48 & 48 \\
Slice thickness (mm) & 4 & 5.5 \\
Distance factor (\%) & 0 & 0 \\
Field of view & $400 \times 262$ & $450 \times 450$ \\
Matrix (reconstructed) & $189 \times 384$ & $259 \times 320$ \\
Resolution (mm) & $1.4 \times 1.0 \times 4$ & $1.7 \times 1.4 \times 5.5$ \\
Bandwidth (Hz/pixel) & 592 & 710 \\
Echo train length & 1 & 256 \\
Parallel imaging & GRAPPA 2 & GRAPPA 2 \\
TA (s) & $5.7(\times 4)^{\mathrm{a}}$ & $8.1(\times 7)^{\mathrm{a}}$
\end{tabular}

T2-HASTE, Half-Fourier acquisition single-shot turbo spin echo; T1-VIBE, T1-weighted Volumetric Interpolated 3D Gradient Echo.

${ }^{a}$ TA measured during single breath-hold. 
ECG-triggering was used to synchronise MR signal acquisition to the cardiac cycle by real-time R-wave detection. However, ECG-triggering is only effective when images were acquired during the diastolic phase with minimal pulsatile motion. Effective ECG-triggering in diastole was not possible within the desired acquisition time for T2-HASTE and fat-suppressed T1-VIBE using spectral adiabatic inversion recovery (SPAIR), and thus has not been evaluated for these sequence-variants.

\section{Data analysis}

\section{Quantitative image analysis}

Volumes-of-interest (VOIs) were established by delineating mediastinal structures (i.e. adipose tissue, muscles, bone, oesophagus, lung, and liver) and tumorous lesions. All VOIs had the same size of $1.6 \mathrm{~cm}^{3}$ and were placed over three slices around the level of the carina. To quantify $\mathrm{IQ}$, signal-to-noise ratio (SNR) and contrast ratio relative to the muscle (CR) were calculated using Eqs (1 and $2)$, respectively.

$$
\begin{gathered}
\mathrm{SNR}=\frac{\mathrm{SI}_{\text {tissue }}}{0.655 \times \mathrm{SD}_{\text {noise }}}, \\
\mathrm{CR}=\frac{\mathrm{SI}_{\text {tissue }}}{\mathrm{SI}_{\text {muscle }}},
\end{gathered}
$$

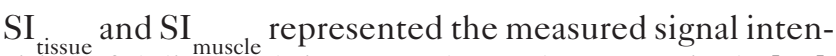
sities of delineated tissues and muscle, respectively [12]. The Rayleigh-distribution in noise outside the body was accounted for in the $\mathrm{SD}\left(\mathrm{SD}_{\text {noise }}\right)$ [12]. Tissue visibility and contrast between adjacent tissues were quantified by differences in $\mathrm{CR}$ of the aforementioned tissues $(\Delta \mathrm{CR})$.

On ${ }^{18} \mathrm{~F}$-FDG-PET/MRIs, ${ }^{18} \mathrm{~F}$-FDG-avid lesions were manually delineated and maximal and mean standard uptake values (SUVs) were obtained. Tumor-to-blood ratios (TBRs) were calculated from ${ }^{18} \mathrm{~F}$-FDG-activity of delineated lesions and aortic blood.

\section{Qualitative image analysis}

Two clinical experts in thoracic oncological imaging (radiologist F.B., and radiation-oncologist M.B.) independently rated the MRI quality using a five-point scoring scale (Supplementary Appendix Table A1, Supplemental digital content 1, http://links.lww.com/NMC/A145). A score of 1 represented poor IQ, not suitable for clinical use, while a score of 5 represented high IQ.

\section{Clinical validation}

\section{Patient compliance and comfort}

Patient compliance was based on the ability to follow and maintain $\mathrm{BH}$ instructions at end-expiration and the ability to remain lying still during scanning. Comfort during scanning was evaluated by enquiring the emotional status of all participants (feelings of stress, claustrophobia, physical discomfort from laying still or acoustic noise) and their perception of scan-time (BH duration, resting periods between $\mathrm{BHs}$, and total scan duration).

\section{Diagnostic performance}

While blinded from clinical data, independent reviewers randomly evaluated the diagnostic value of all ${ }^{18} \mathrm{~F}$-FDGPET/MRI and ${ }^{18} \mathrm{~F}$-FDG-PET/CT images according to the AJCC/UICC TNM-system with corresponding stage grouping (7th ed.). M-stage was evaluated on whole-body PET/CT and on thoracic PET/MRIs. Clinical reports with diagnoses based on PET/CT and histopathologic/ cytopathologic analyses after mediastinoscopy or ultrasound-guided transbronchial needle aspiration were obtained as part of standard clinical care and served as the golden standard for further reference. All PET/MRI analyses were performed using OsiriX software (Pixmeo Sarl, Bernex, Switzerland).

\section{Statistical analysis}

Firstly, for protocol-optimisation statistical differences between volunteer and patient data in SNR of normal tissue was tested using a paired Student $t$-test. Populations were pooled when no significant differences were apparent. Secondly, differences in SNR and CR between sequence-variations were tested for statistical significance using two-way analysis of variance with Tukey correction, respectively. Thirdly, differences in CR between tumour/LN and adjacent tissue were statistically tested per sequence-variation using two-way analysis of variance with Tukey correction to quantify contrast between tissues, and tumour visibility, relative to muscle-tissue. Finally, reader agreement in qualitative image assessment was analysed using weighted $\kappa$ reliability statistics $(\kappa<0.2=$ slight agreement,$\quad \kappa=0.2-0.4=$ fair, $\quad \kappa=0.41-$ $0.6=$ moderate, $\kappa=0.6-0.8=$ good, $\kappa>0.8=$ excellent $)$.

For clinical validation in patients, we first tested the hypothesis that there is no statistically significant difference in $\mathrm{SUV}_{\text {mean }}, \mathrm{SUV}_{\text {max }}$, and TBR acquired on PET/ MRI and PET/CT using a paired Student $t$-test and a Bland-Altman plot. The diagnostic performances of PET/ MR and PET/CT (i.e. TNM staging) were compared in terms of sensitivity and specificity using a McNemar test. All statistical analyses were performed using GraphPad Prism v6.01 (GraphPad, California, USA). $P$ values less than 0.05 were considered statistically significant.

\section{Results \\ MR-protocol optimisation for mediastinal imaging Quantitative image analyses}

For T1-VIBE and T2-HASTE, SNRs of healthy participants and patients were not significantly different $(P=0.52$ and 0.065$)$. In tumorous lesions, SNR was only significantly different between T1-VIBE BH with and without ECG-triggering $(P=0.030)$. In ${ }^{18} \mathrm{~F}-\mathrm{FDG}$ avid $\mathrm{LN}$, no significant difference was found between T1-VIBE sequence-variants $(P>0.05)$. In the pooled population of volunteer and patient data, there were no statistically significant differences between T1-VIBE sequences acquired in $\mathrm{BH}$ and $\mathrm{FB}(P>0.05)$. However, the addition of ECG-triggering to either T1-VIBE BH or 
FB sequence-variations resulted in significantly greater SNR for all soft-tissue structures of the mediastinum $(P \leq 0.001)$. However, ECG-triggering not significantly affect the SNR of bone structures $(P=0.76$ and 0.73$)$ and lung tissue ( $P \geq 0.99$ and $P=0.998)$, respectively (Fig. 1a). The addition of SPAIR to T1-VIBE BH or T1-VIBE FB did not result in statistically significant differences in SNR $(P>0.1)$.

In T2-HASTE images, SNR was unaffected by $\mathrm{BH}$ compared to free breathing for all tissues $(P>0.97)$. The addition of SPAIR fat-suppression to T2-HASTE BH and T2-HASTE FB significantly reduced quantitative SNR in adipose tissue $(P \leq 0.0001$ and $\leq 0.0001)$ and bone $(P=0.0236$ and 0.0197$)$, but remained unaffected for all other tissues. These findings concerning the SNRs of various tissues for each sequence-variation are presented in Supplementary Appendix Fig. A1 (Supplemental digital content 2, http://links.lwwe.com/NMC/A146).

Contrast within images indicated the ability to differentiate various tissues from SI ${ }_{\text {muscle }}$ (Fig. 1). CR-values were not significantly different between T1-VIBE and between T2-HASTE sequence-variants $(P>0.9)$, except between sequences acquired with and without SPAIR fat-suppression $(P<0.0001)$. However, within T1-VIBE sequence-variants contrast between adjacent tissues $(\triangle \mathrm{CR})$ was significant between oesophagus, lung, and fat $(P \leq 0.0001)$, between lung, bone, and liver $(P \leq 0.0001)$, and between tumour and fat $(P \leq 0.0001)$. In ECGtriggered T1-VIBE sequences, $\triangle \mathrm{CR}$ was also significant between oesophagus and bone $(P<0.05)$, and between tumour and lung $(P<0.05)$. The addition of SPAIR did not result in additional significant results of $\triangle \mathrm{CR}$. For T2-HASTE BH and T2-HASTE FB, $\triangle \mathrm{CR}$ was significant between lung, oesophagus $(P=0.013$ and 0.008$)$, and bone $(P=0.0008$ and 0.0012$)$ and between fat and all other tissues $(P \leq 0.0001)$. When fat was suppressed by SPAIR, no significant differences in contrast were observed. In addition, differences in SNR and CR can be appreciated and the visibility of mediastinal tissues and cancerous lesions can be deduced for each T1-VIBE sequence-variant (Fig. 1). Tumour visibility was highest in ECG-triggered T1-VIBE sequences acquired in BH ( $\mathrm{SNR}=62.6, \mathrm{CR}=0.86)$, followed closely by $\mathrm{T} 1-\mathrm{VIBE}$ $\mathrm{ECG} \mathrm{FB}(\mathrm{SNR}=59.2, \mathrm{GR}=0.83)$. However, the overall IQ and visibility of all mediastinal structures are determined by the balance of SNR and $\triangle \mathrm{CR}$, which is demonstrated by the spread between data-points and the slope of the plots in Fig. 1. Visibility of all mediastinal structures was lower in T1-VIBE sequences acquired without ECG-triggering and further decreased in fat-suppressed sequences, which presented more clustering of datapoints and a lower slope. For T2-HASTE sequences, an acceptable distribution of SNR-CR data points was noted (Supplementary Appendix Fig. A1, Supplemental digital content 2, http://links.lww.com/NMC/A146). However, no differences between sequence-variants were evident.

\section{Qualitative image analyses}

T1-VIBE and T2-HASTE IQ showed good inter-reader agreement $(\kappa=0.617$ and 0.625 , respectively; Table 2 and Fig. 2). For T1-VIBE, qualitative IQ was scored highest for images acquired during $\mathrm{BH}$ and with ECG-triggering and contained a homogeneous distribution of SI within VOIs and visually less noise. T1-VIBE BH ECG showed clear organ-edge detection with low incidences of artefacts at the mediastinum. In FB ECG-triggered T1-VIBE images more motion artefacts were present at the mediastinum, leading to qualitatively moderate IQ.

Generally, the IQ of T2-HASTE images was scored lower than T1-VIBE images. Moderate IQ was registered for both T2-HASTE BH and FB sequence-variants with moderate contouring of mediastinal structures. However, a notable increase in IQ of T2-HASTE was perceived towards abdominal regions (e.g. gastroesophageal junction, liver).

For both T1-VIBE and T2-HASTE, SPAIR adequately suppressed fat near the mediastinum. However, the addition of SPAIR visually increased image noise and led to poor IQ, making it difficult to detect and differentiate mediastinal structures.

\section{Clinical validation}

\section{Patient compliance and comfort}

All included participants successfully underwent PET/ MRI and were able to maintain BHs at end-expiration for all sequences (Table 3). However, patients with mediastinal malignancies had more trouble complying to scanning instructions and reported that the repetition frequency of multiple $\mathrm{BHs}$ made it increasingly difficult to consistently maintain $\mathrm{BH}$. The $\mathrm{BH}$-duration of $8.7 \mathrm{~s}$ during T2-HASTE image acquisition was perceived as nearing the patients' limits. Longer BHs, more $\mathrm{BH}$ repetitions, or the use of single BH schemes are therefore unlikely to be successful in patients with mediastinal malignancies. After optimisation, the total scan duration was $15-20 \mathrm{~min}$ and no excessive physical or psychological discomfort was reported.

\section{PET-image quality}

The radioactive dose at the start of ${ }^{18} \mathrm{~F}$-FDG-PET/CT was $105 \pm 33 \mathrm{MBq}$ at $65 \pm 10 \mathrm{~min}$ postinjection and $71 \pm 21$ $\mathrm{MBq}$ at the start of ${ }^{18} \mathrm{~F}$-FDG-PET/MR $(127 \pm 19 \mathrm{~min}$ postinjection) (Table 3). Despite different attenuation-correction methods, no significant differences were found between modalities in $\mathrm{SUV}_{\text {mean }}$ and $\mathrm{SUV}_{\max }$ for ${ }^{18}$ F-FDG-avid tumours $(P=0.736$ and 0.177$)$ and suspicious LNs $(P=0.184$ and 0.535$)$, respectively. Likewise, TBR-values of PET/MR was not significantly different from PET/CT for ${ }^{18} \mathrm{~F}-\mathrm{FDG}$-avid tumours $(P=0.082)$ and suspicious LNs $(P=0.125)$. Potentially, the delay in injection time was compensated by the extended PETacquisition time for the PET/MR protocol compared to the clinical PET/CT protocol. 
Fig. 1
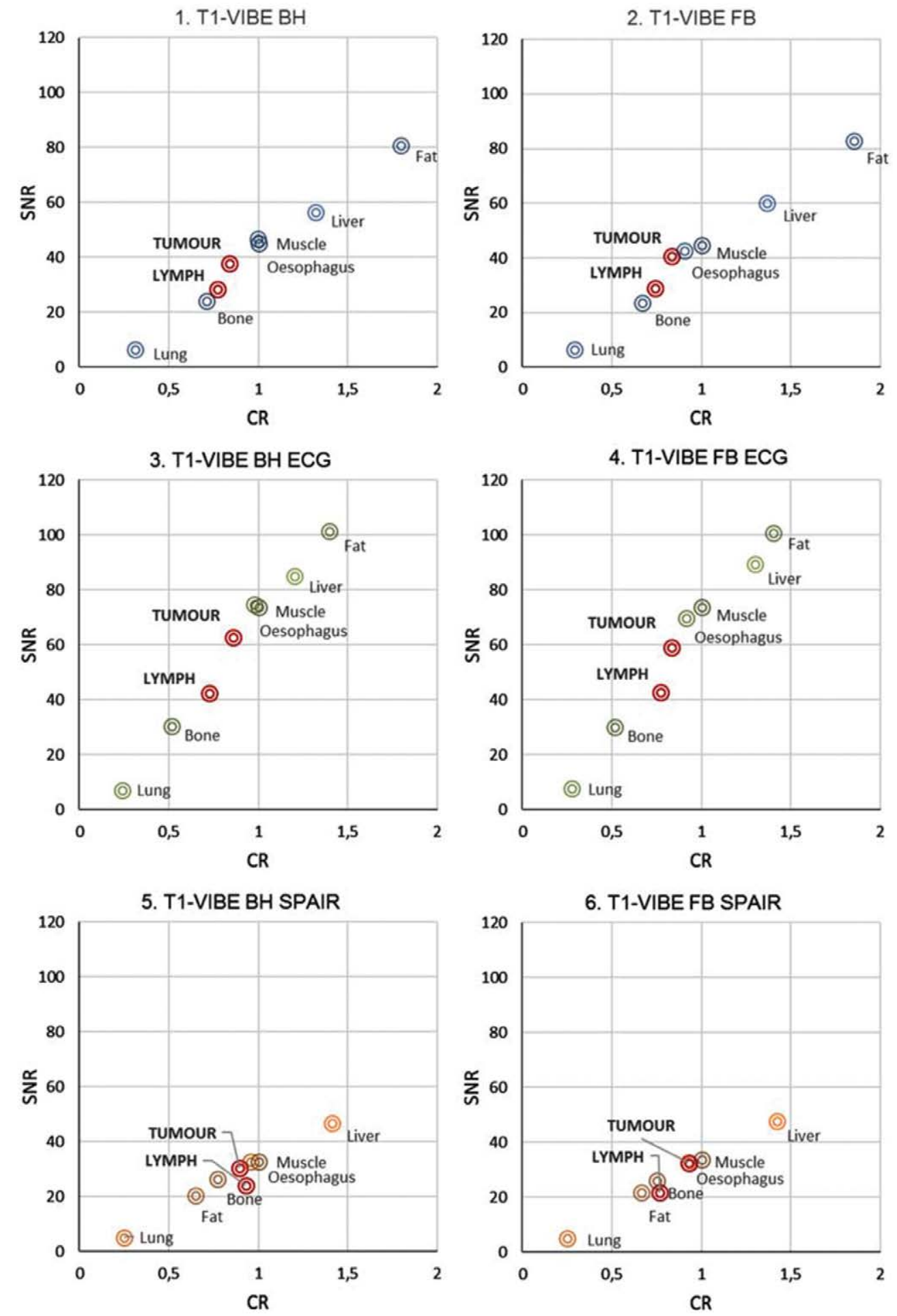

Quantitative analyses of T1-VIBE image quality acquired in cancer patients ( $n=10$, eight primary tumours, five LN metastases) by assessment of signal-to-noise ratio (SNR) and contrast ratio (CR) per sequence-variant over different tissues. VOls were drawn over target regions and background-regions located outside the body. LN, lymph node; T2-HASTE, Half-Fourier acquisition single-shot turbo spin echo; T1-VIBE, T1-weighted Volumetric Interpolated 3D Gradient Echo. 
Table 2 Qualitative assessment of MRI quality assessed in healthy volunteers and patients using a five-point scoring scale (score $1=$ low image quality, score $5=$ high image quality)

Qualitative five-point scoring $^{\mathrm{a}}$

\begin{tabular}{lccc} 
Sequence & $R_{1}$ & $R_{2}$ & $\kappa$ \\
\hline T1-VIBE & & & 0.617 \\
BH & $2 \pm 0.5$ & $3 \pm 0.4$ & \\
BH-ECG & $4 \pm 0.5$ & $4 \pm 0.3$ & \\
BH-SPAIR & $2 \pm 0.5$ & $2 \pm 0.5$ & 0.625 \\
FB & $2 \pm 0.5$ & $3 \pm 0.5$ & \\
FB-ECG & $3 \pm 0.0$ & $3 \pm 0.3$ & \\
FB-SPAIR & $2 \pm 0.6$ & $2 \pm 0.5$ & \\
T2-HASTE & & & \\
BH & $3 \pm 0.5$ & $3 \pm 0.3$ & \\
BH-SPAIR & $2 \pm 0.3$ & $3 \pm 0.3$ & \\
FB & $2 \pm 0.6$ & $2 \pm 0.3$ & \\
FB-SPAIR & $2 \pm 0.4$ & & \\
\hline
\end{tabular}

$\mathrm{BH}$, breath-hold; FB, free-breathing; SPAIR, spectral adiabatic inversion recovery; T2-HASTE, Half-Fourier acquisition single-shot turbo spin echo; T1-VIBE, T1-weighted Volumetric Interpolated 3D Gradient Echo.

${ }^{a}$ Qualitative five-point scoring system is presented in Supplementary Appendix Table A2 (Supplemental digital content 1, http://links./ww.com/NMC/A145).

Subjective assessment of PET-images indicated clinically-acceptable IQ and excellent alignment with the anatomical substrate on MRI and CT, respectively. Generally, lesions could be well differentiated with slightly blurred lesion edges on PET/MRIs. In patients 3 and 4, considerable blurring resulted in the uncertain definition of ${ }^{18}$ F-FDG-avidity and lesion differentiation.

\section{Diagnostic performance}

No significant differences in sensitivity and specificity on defining T-stage $(P>0.99)$ or N-stage $(P>0.99)$ was presented between PET/MR and PET/CT, compared with histopathological/cytopathological analyses. However, T-status was more often over-staged on ${ }^{18} \mathrm{~F}$-FDG-PET/ CT (3/10 patients) and N-status was more frequently under-staged on ${ }^{18} \mathrm{~F}$-FDG-PET/MR (2/5 patients) (Table 4 and Figs 3 and 4). In patient 5 and 7, M-status were accurately evaluated on both whole-body ${ }^{18} \mathrm{~F}$-FDGPET/CT and thorax ${ }^{18}$ F-FDG-PET/MRIs.

\section{Discussion}

In this study, we presented a clinically-manageable, region-specific PET/MR protocol with high patient compliance and stable MR pulse sequences that consistently produce images of high IQ and diagnostic accuracy, within a reasonable acquisition time. This carefully-optimised PET/MR protocol has led to less over-staging than $\mathrm{PET} / \mathrm{C}$ T alone and establishes an important region-specific use in the patient workflow.

Based on qualitative and quantitative image analyses, T1-VIBE and T2-HASTE were adequate sequences for mediastinal MRI and tumour visualisation. For BH or FB T1-VIBE, no significant differences were found in SNR and CR between images acquired with or without SPAIR. Although SPAIR performed well at fat suppression in all sequences, organ-edges became blurred which made visually differentiating mediastinal structures more difficult. In contrast, the addition of ECGtriggering to either $\mathrm{BH}$-variant or $\mathrm{FB}$-variant resulted in a significant increase in quantitative $I Q$, indicating that ECG-triggering had the most impact on IQ by reducing the effect of cardiac and aortic motion during scanning. As such, ECG-triggered T1-VIBE presented high contrast between adjacent tissues and ease of differentiating tumours and metastatic LNs, relative to muscle. The application of ECG-triggered T1-VIBE could be especially useful in assessing mediastinal tumours with infiltrates into the pericardium or large vessels [11]. However, ECG-triggering might be inefficient in patients with arrhythmia or complex QRS-patterns.

Qualitative assessment of ECG-triggered T1-VIBE indicated a slight preference for $\mathrm{BH}$-sequences as fewer image-distorting MR-artefacts were present at the mediastinum. By maintaining $\mathrm{BH}$ at end-expiration, mean diaphragm motion is more stable $(0.15 \mathrm{~mm} / \mathrm{s})$ compared to end-inspiration $\mathrm{BH}(0.1-7.9 \mathrm{~mm} / \mathrm{s})$ [13]. This recommendation is in contrast to the suggested protocol of Biederer et al. $[11,14]$ that contain end-inspiration $\mathrm{BH}$ instructions of $20 \mathrm{~s}$. However, ample time between BH-intervals is needed to avoid fatigue and failure to maintain reproducible BHs. Therefore, short, multiple BHs are preferred without tiring the patient. When a patient is unfit to perform these BHs, a FB ECG-triggered T1-VIBE could still produce adequate images for clinical use. In this, however, motion artefacts would appear more frequent. Alternatively, the respiratory motion could be handled by automated respiratory triggering or gated acquisition using a navigator sequence to monitor the diaphragm's position [15-17]. However, these techniques are not flawless in compensating motion artefacts and will extend the total scan duration [11]. In literature, T1-VIBE has been shown to be robust in the presence of cardiac pulsation, able to identify small pulmonary nodules, and has been promoted for thoracic imaging $[8,10,18]$. For T2-HASTE, quantitative and qualitative IQ was unaffected by acquisition in $\mathrm{BH}$ or $\mathrm{FB}$. However, lengthy $\mathrm{BH}$-durations were not patient friendly and prolonged acquisition-time. T2-HASTE acquisition in FB might, therefore, be preferred in clinical practice. Previous studies recommended applying fat saturation in contrast-enhanced MRI to differentiate mediastinal LNs from the surrounding fat $[10,11,19]$. However, in this study, no contrast agent was used and the addition of SPAIR did not significantly affect SNR and contrast between tumours, LN, and surrounding tissue. Nevertheless, unenhanced T2-HASTE sequences have been reported to sensitively detect pleural effusion and intra-atelectatic tumour growth [9,20,21]. The effect of motion and MR motion-correction was less prone on PET-images, although motion has a negative effect on the localisation of PET-signal.

In this study, the diagnostic performance of ${ }^{18} \mathrm{~F}-\mathrm{FDG}$ PET/MR was not inferior to ${ }^{18} \mathrm{~F}-\mathrm{FDG}-\mathrm{PET} / \mathrm{CT}$ in 
Fig. 2

(a)

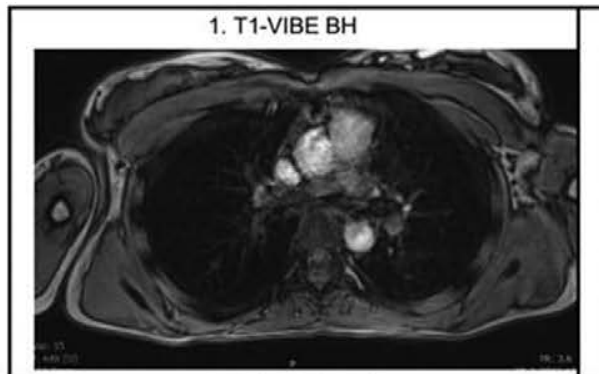

3. T1-VIBE BH ECG

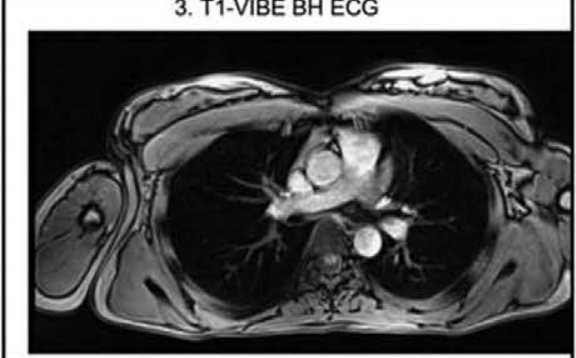

5. T1-VIBE BH SPAIR

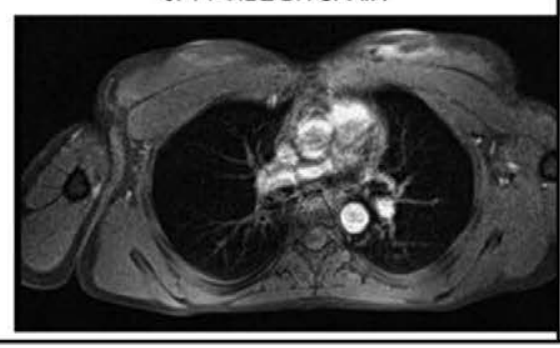

(b)

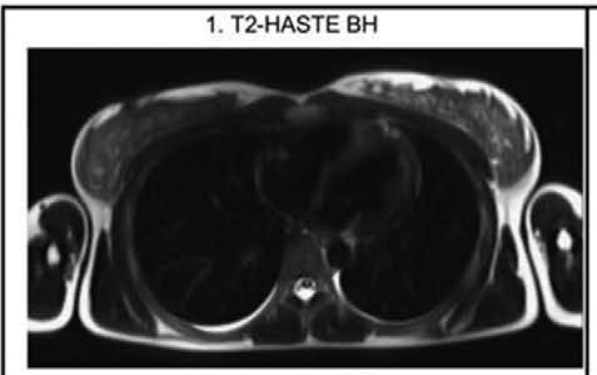

3. T2-HASTE BH SPAIR

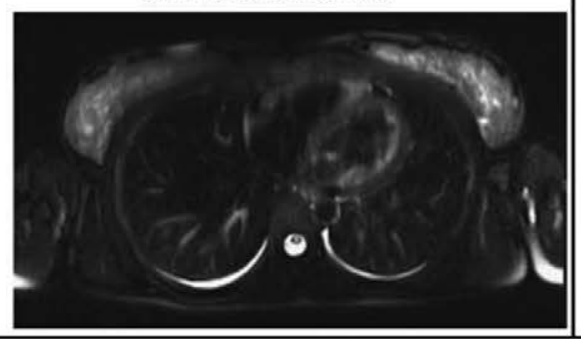

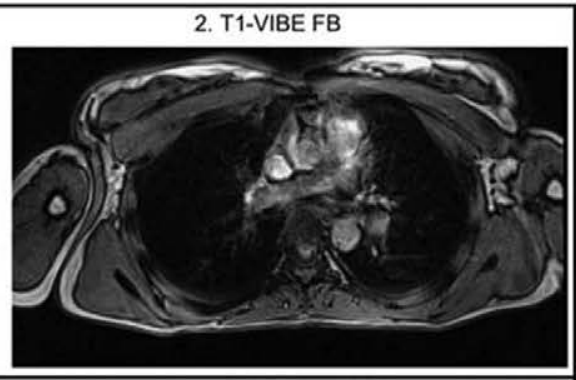

4. T1-VIBE FB ECG

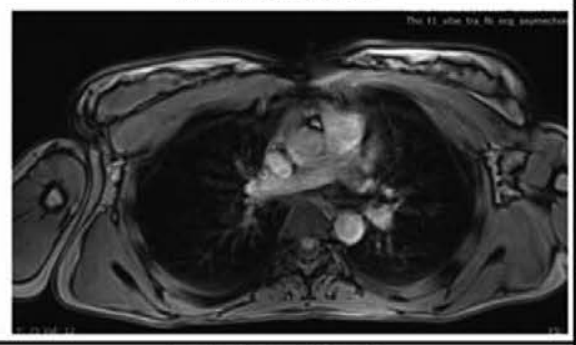

6. T1-VIBE FB SPAIR

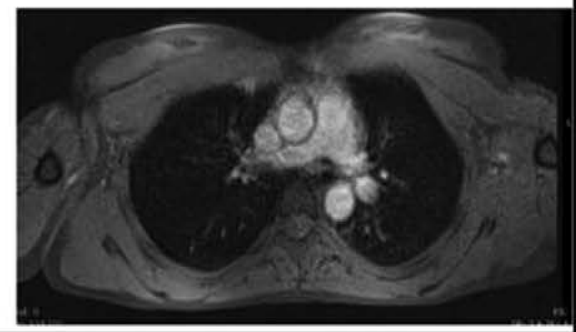

2. T2-HASTE FB

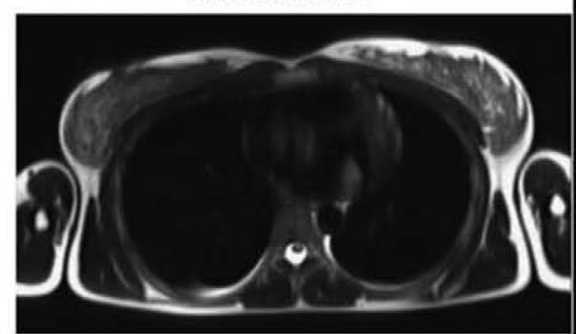

4. T2-HASTE FB SPAIR

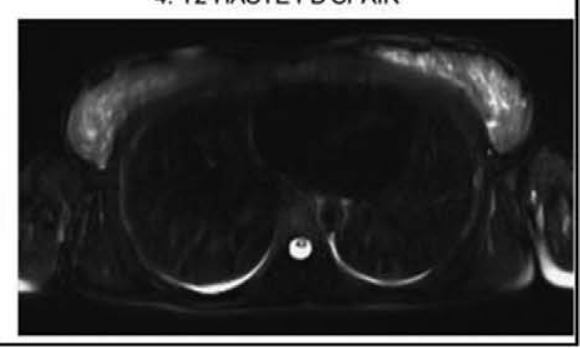

T1-VIBE (a) and T2-HASTE (b) images acquired in healthy volunteers at varying patient-specific parameters [in breath hold (BH), in free-breathing (FB), and with/without ECG-triggering, or SPAIR fat suppression]. T2-HASTE, Half-Fourier acquisition single-shot turbo spin echo; T1-VIBE, T1-weighted Volumetric Interpolated 3D Gradient Echo.

defining tumour or LN status. SUV $\mathrm{Smen}_{\text {and }}$ SUV were not significantly different between ${ }^{\text {mepr }}$ F-FDG-PET/ MR and ${ }^{18} \mathrm{~F}$-FDG-PET/CT meaning that the measured
PET-values were not affected by the applied attenuation correction technique for each modality, respectively. In T-staging, pleural infiltration of NSCLC (Fig. 4) and 
Table 3 Patient information and quantitative analyses of ${ }^{18} \mathrm{~F}-\mathrm{FDG}-\mathrm{PET}$ image quality of both PET/CT and PET/MRI modalities

\begin{tabular}{|c|c|c|c|c|c|c|c|c|}
\hline \multirow[b]{2}{*}{ Patient } & \multirow[b]{2}{*}{${ }^{18} \mathrm{~F}-\mathrm{FDG}$ dose $(\mathrm{MBq})$} & \multirow[b]{2}{*}{ Corrected dose $(\mathrm{MBq})$} & \multicolumn{3}{|c|}{ Primary tumour } & \multicolumn{3}{|c|}{ Lymph node } \\
\hline & & & SUV $_{\max }$ & $\mathrm{SUV}_{\text {mean }} \pm \mathrm{SD}$ & TBR & SUV $_{\max }$ & $\mathrm{SUV}_{\text {mean }} \pm \mathrm{SD}$ & TBR \\
\hline \multicolumn{9}{|l|}{ PET/CT } \\
\hline 1 & 234.2 & 153 & 9.0 & $5.1 \pm 1.9$ & 2.8 & - & - & - \\
\hline 2 & 152.4 & 98 & 0.8 & $0.6 \pm 0.1$ & 0.4 & - & - & - \\
\hline 3 & 164.1 & 110 & 4.1 & $2.4 \pm 0.9$ & 1.6 & - & - & - \\
\hline 4 & 116 & 68 & 1.0 & $0.7 \pm 0.2$ & 0.5 & 2.2 & $1.6 \pm 0.4$ & 1.2 \\
\hline 5 & 208.9 & 150 & 13.8 & $10.2 \pm 2.2$ & 5.3 & 5.6 & $3.5 \pm 1.2$ & 1.8 \\
\hline 6 & 163.3 & 70 & No tumour & - & - & - & - & - \\
\hline 7 & 139.75 & 98 & 9.0 & $7.7 \pm 0.7$ & 4.4 & 8.3 & $5.0 \pm 1.5$ & 2.2 \\
\hline 8 & 142.4 & 97 & No tumour & - & - & - & - & - \\
\hline 9 & 147.8 & 100 & 25.2 & $16.2 \pm 3.3$ & 13.9 & 2.9 & $2.0 \pm 0.5$ & 1.7 \\
\hline 10 & 150.1 & 63 & 9.7 & $4.6 \pm 2.1$ & 4.1 & 4.9 & $3.3 \pm 0.9$ & 2.9 \\
\hline \multicolumn{9}{|c|}{ PET/MR } \\
\hline 1 & 234.2 & 116 & 13.0 & $6.8 \pm 2.6$ & 4.5 & - & - & - \\
\hline 2 & 152.4 & 59 & 0.9 & $0.6 \pm 0.15$ & 0.6 & - & - & - \\
\hline 3 & 164.1 & 71 & 6.8 & $3.5 \pm 1.5$ & 2.1 & - & - & - \\
\hline 4 & 116 & 50 & 1.0 & $0.5 \pm 0.2$ & 0.6 & 2.7 & $1.6 \pm 0.6$ & 1.9 \\
\hline 5 & 208.9 & 100 & 21.2 & $9.7 \pm 5.5$ & 5.9 & 6.5 & $4.2 \pm 1.4$ & 2.5 \\
\hline 6 & 163.3 & 55 & No tumour & - & - & - & - & - \\
\hline 7 & 139.75 & 65 & 10.5 & $7.41 \pm 1.28$ & 1.3 & 7.8 & $4.8 \pm 1.3$ & 4.3 \\
\hline 8 & 142.4 & 58 & No tumour & - & - & - & - & - \\
\hline 9 & 147.8 & 73 & 24.1 & $17.2 \pm 3.2$ & 20.1 & 2.9 & $2.0 \pm 0.5$ & 2.7 \\
\hline 10 & 150.1 & 59 & 7.6 & $3.5 \pm 1.8$ & 3.3 & 2.6 & $2.2 \pm 0.4$ & 2.1 \\
\hline
\end{tabular}

Maximal and mean standardized uptake value $\left(\mathrm{SUV}_{\max }\right.$ and $\mathrm{SUV}_{\text {mean }}$ ) were measured over tumour regions. Tumour-to-blood uptake ratio (TBR) was based on SUV of $_{\text {mean }}$ tumour regions and venous blood pools.

$\mathrm{CT}$, computed tomography; ${ }^{18} \mathrm{~F}-\mathrm{FDG}$, fluorine-18-fluorodeoxyglucose.

Table 4 Patient information and diagnostic performance (pathological and clinical TNM staging) based on PET/CT and PET/MRI

\begin{tabular}{|c|c|c|c|c|c|}
\hline \multirow[b]{2}{*}{ Patients no. } & \multirow[b]{2}{*}{ Cancer } & \multirow[b]{2}{*}{ Sex } & \multirow[b]{2}{*}{ Age } & \multicolumn{2}{|c|}{ Diagnosis } \\
\hline & & & & ${ }^{18}$ F-FDG-PET/CT & Clinical report \\
\hline 1 & EC & Male & 80 & pT1No & cT2NOMO \\
\hline 2 & NSCLC & Female & 54 & PTONO & cT1aNOMO \\
\hline 3 & NSCLC & Male & 64 & pTxNO & cTONOMO \\
\hline 4 & NSCLC & Male & 41 & pTxNO & cTONO \\
\hline 5 & NSCLC & Male & 70 & pT4N2M1 & cT2bN2M1a \\
\hline 6 & NSCLC & Female & 74 & pTONO & cTONO \\
\hline 7 & NSCLC & Male & 71 & pT4N3M1 & cT4N3M1b \\
\hline 8 & NSCLC & Female & 52 & PTONO & cTONO \\
\hline 9 & NSCLC & Male & 63 & pT4N0-2 & cT4N2MO \\
\hline 10 & EC & Male & 70 & pTxN1 & cT3N1M0 \\
\hline Patients no. & Cancer & Sex & Age & ${ }^{18}$ F-FDG-PET/MR & Clinical Report \\
\hline 1 & EC & Male & 80 & pT2NO & cT2NOMO \\
\hline 2 & NSCLC & Female & 54 & pT1NO & cT1aNOMO \\
\hline 3 & NSCLC & Male & 64 & pTxNO & cTONOMO \\
\hline 4 & NSCLC & Male & 41 & pTxN2 & cTONO \\
\hline 5 & NSCLC & Male & 70 & pT2N1M1 & cT2bN2M1a \\
\hline 6 & NSCLC & Female & 74 & PTONO & cTONO \\
\hline 7 & NSCLC & Male & 71 & pT4N3M1 & cT4N3M1b \\
\hline 8 & NSCLC & Female & 52 & pTONO & cTONO \\
\hline 9 & NSCLC & Male & 63 & pT3N1 & cT4N2MO \\
\hline 10 & $\mathrm{EC}$ & Male & 70 & pT2N1 & cT3N1M0 \\
\hline
\end{tabular}

CT, computed tomography; ${ }^{18}$ F-FDG, fluorine-18-fluorodeoxyglucose; NSCLC, non-small-cell lung cancer.

thickening of oesophageal wall layers in EC (Fig. 3c-f) could be better detected on high-resolution MRI, resulting in less over-staging compared to ${ }^{18} \mathrm{~F}$-FDG-PET/ CT (Fig. 3a and b) $[3,15,22]$. In N-staging, contradicting results of diagnostic performance have been reported in single-injection ${ }^{18} \mathrm{~F}$-FDG-PET/CT versus ${ }^{18} \mathrm{~F}$-FDGPET/MR studies. In EG, Lee et al. [22] showed that
PET/MR presented the highest diagnostic performance for T-staging, followed by EUS, PET/CT and CT. In agreement to our study, however, these authors demonstrated no significant differences in diagnostic accuracy for diagnosing nodal metastasis. In NSCLC, no clear (dis) advantage of ${ }^{18} \mathrm{~F}$-FDG-PET/MR over ${ }^{18} \mathrm{~F}$-FDG-PET/ CT is determined in TNM-staging or therapeutic decision-making [23-25]. Heusch et al. [26] showed no difference in T-staging or N-staging NSCLC with sensitivity, specificity, and accuracy $(P=0.48)$. However, fewer over/ under-staging (9\%) were discovered using ${ }^{18} \mathrm{~F}$-FDGPET/MR, which concurred to our findings. In our study, ${ }^{18} \mathrm{~F}$-FDG-PET/MR produces clinically acceptable images from simultaneously acquired PET and MR-data of similar value as the clinically standard ${ }^{18} \mathrm{~F}$-FDG-PET/ CT [24,26,27]. In addition, ${ }^{18} \mathrm{~F}$-FDG-PET/MRIs could be clinically valuable by detecting tumour infiltration in adjacent organs. However, the impact of mediastinal-specific ${ }^{18}$ F-FDG-PET/MRI on TNM-staging needs to be investigated further in large-scale studies.

This study is limited in patient population and in the diversity of MR-sequences tested. We selected conventional, clinical T1-weighted and T2-weighted sequences and did not alter sequence-parameter settings while testing the aforementioned sequence-variables. In some patients, the gastroesophageal junction was not depicted due to the limited field-of-view of T1-VIBE. For a future depiction of EG, cranio-caudal field-of-view-settings need to be adjusted to extent from commonly affected cervical to coeliac LN-stations in EC. Alternative sequences to T1-VIBE (radial-VIBE, CAIPIRINHAVIBE, contrast-enhanced VIBE) have been previously 
Fig. 3

(a)

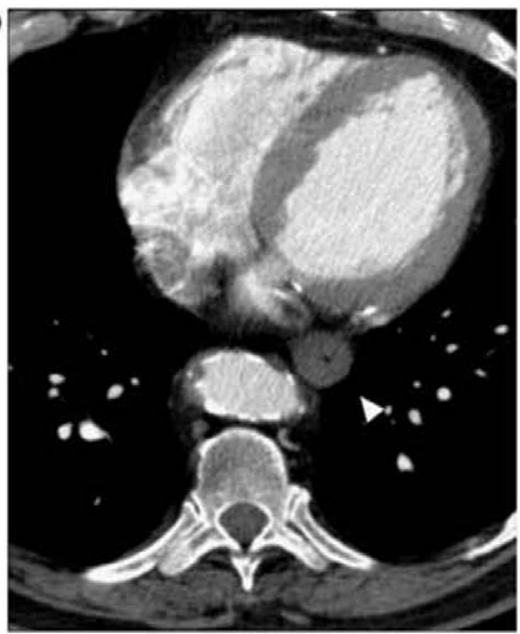

(c)

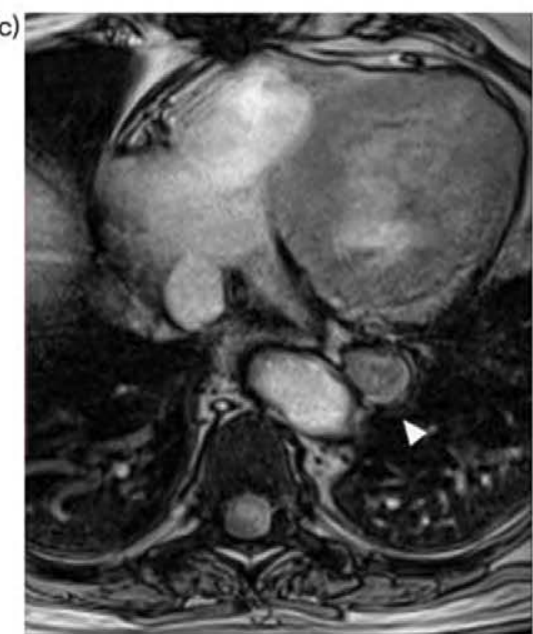

(e) (b)

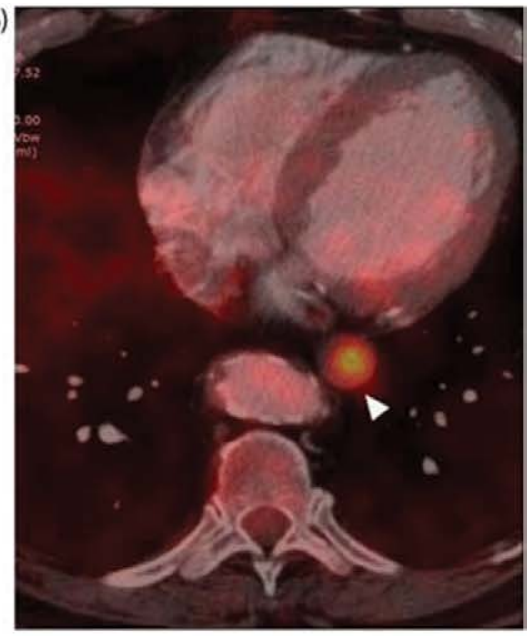

(d)

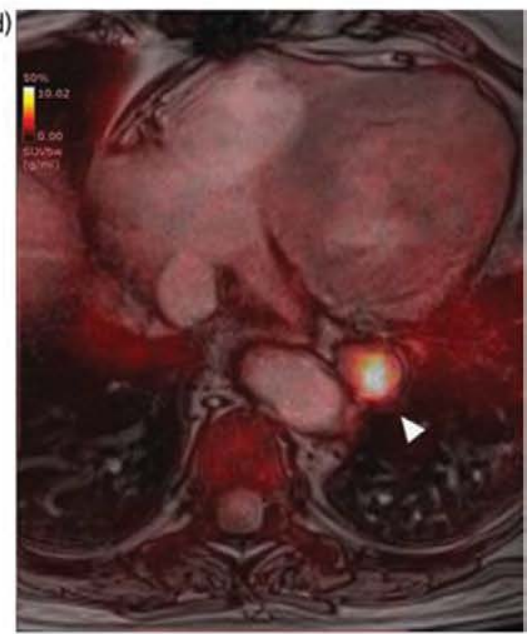

(f)

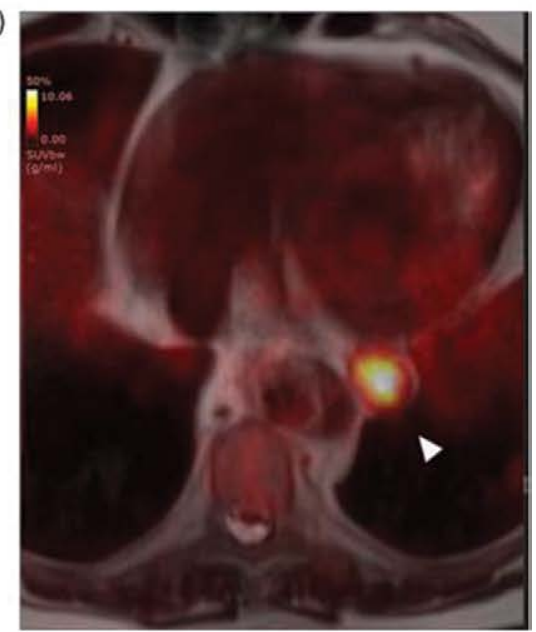

${ }^{18}$ F-FDG-PET/CT $(a, b)$ and ${ }^{18}$ F-FDG-PET/MRI (c-f) of patient 1 (male, 68 years) with oesophageal cancer (white triangle). Fused images (b, $\left.d, f\right)$ are respectively compiled of thoracic CT (a), T1-VIBE BH ECG (c), and T2-HASTE BH (e). BH, breath hold; CT, computed tomography; ${ }^{18}$ F-FDG, fluorine-18-fluorodeoxyglucose; T2-HASTE, Half-Fourier acquisition single-shot turbo spin echo; T1-VIBE, T1-weighted Volumetric Interpolated 3D Gradient Echo. 
Fig. 4

(a)

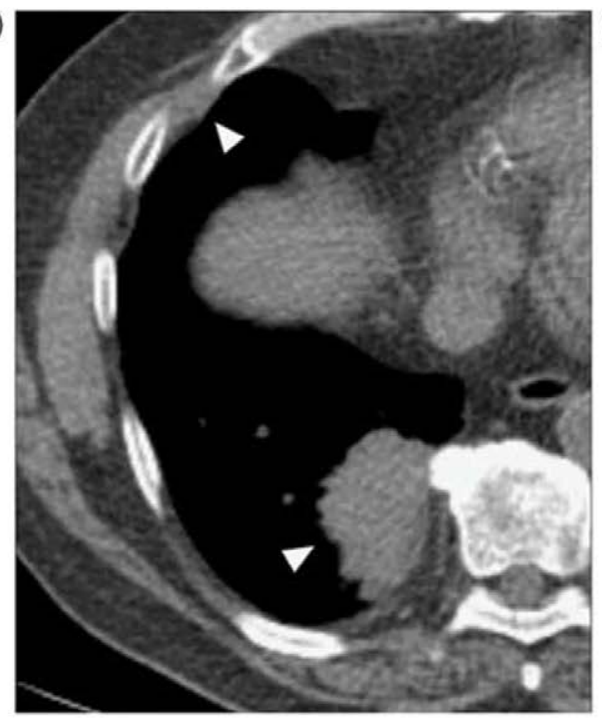

(b)

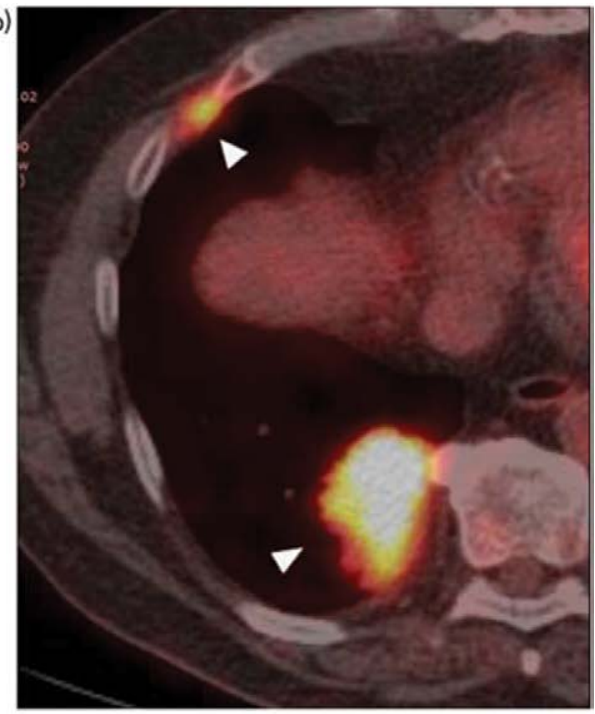

(c)

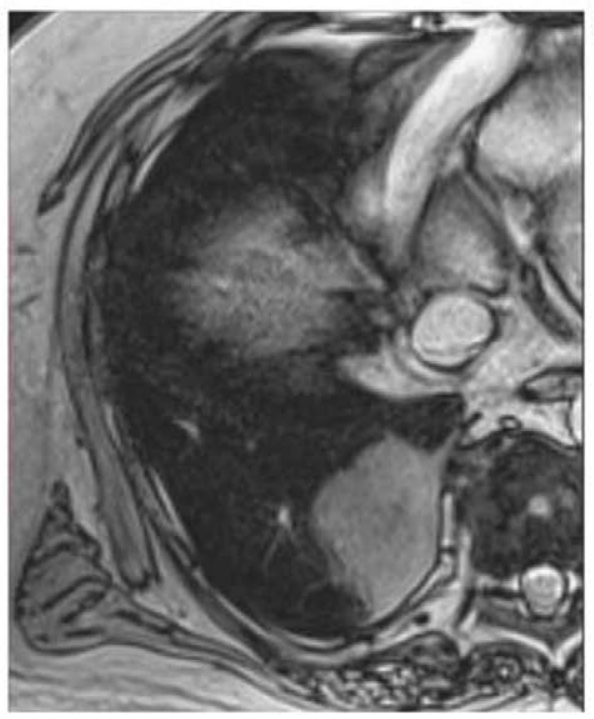

(d)

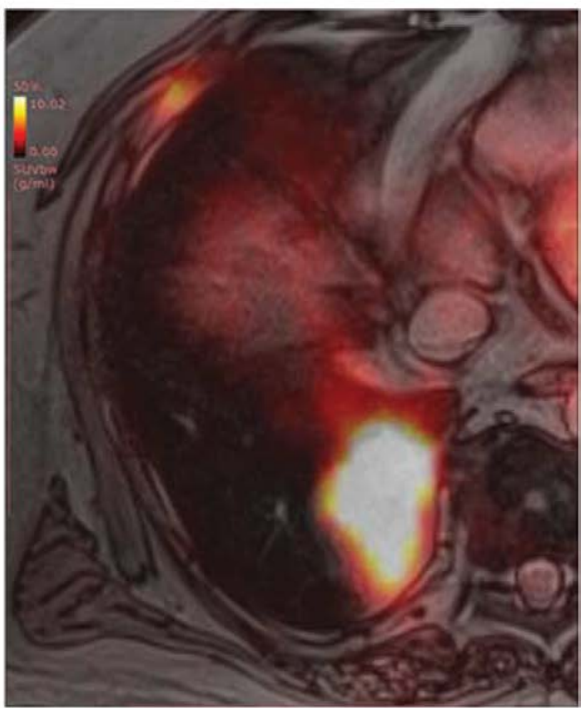

${ }^{18} \mathrm{~F}$-FDG-PET/CT $(\mathrm{a}, \mathrm{b})$ and ${ }^{18} \mathrm{~F}$-FDG-PET/MRI (c, d) of patient 5 (male, 70 years) with non-small-cell lung cancer (NSCLC) and thoracic metastases (white triangles). Tumour infiltration was dismissed on ECG-triggered T1-VIBE images acquired in breath-hold (b). ${ }^{18} \mathrm{~F}-\mathrm{FDG}$, fluorine-18-fluorodeoxyglucose; T1-VIBE, T1-weighted Volumetric Interpolated 3D Gradient Echo.

studied and could potentially improve mediastinal imaging [28]. Radial-VIBE is less susceptible to respiratory-motion artefacts and showed high overall IQ in paediatric abdominal MRI [29]. However, this experimental sequence is prone to streaking artefacts and not standardly-available on PET/MR-systems [30]. CAIPIRINHAVIBE has been shown to produce abdominal and thoracic images with higher SNR and spatial resolution compared to T1-VIBE by improving parallel imaging and reducing aliasing artefacts [31].

For successful clinical imaging, PET/MR protocols need to be developed for time-efficient, region-specific acquisition of high-quality images [7,32]. Efficient PET/ MR protocols could be expanded with DW MRI, steadystate free precession or inversion recovery sequences that have proven to be clinically useful in EC and NSCLC $[10,33,34]$. However, several studies suggest an overlap in metabolic activity and tumour-cellularity as assessed by ${ }^{18}$ F-FDG-PET and DW-MRI, respectively $[35,36]$. Acquisition of time-consuming DW-MRI is only worthwhile when truly synergistic information could be gained for either diagnostics or treatment-decision making $[6,37]$. Therefore, efficient PET/MR protocols may need to combine anatomical and functional MRI with PET-imaging 
that uses radioactive tracers beyond ${ }^{18} \mathrm{~F}$-FDG, such as ${ }^{18} \mathrm{~F}$-HX4 (i.e. hypoxia imaging), but reserve ${ }^{18} \mathrm{~F}$-FDG (i.e. glucose metabolism) for PET/CT that is already well-integrated in clinical practice and holds great diagnostic value. In radiation therapy, PET/MRIs could be valuable in precisely differentiating malignant lesions from organsat-risk, defining the metabolic phenotype of mediastinal tumours, and defining treatment strategies. The search for key applications of PET/MR needs to be further stimulated to work towards personalised patient care [38-41].

\section{Conclusion}

A region-specific PET/MRI protocol, including T1-VIBE ECG and T2-HASTE, can be used for dedicated mediastinal examinations and produce images with acceptable quality that is both patient-friendly and potentially clinically valuable in thoracic oncology.

\section{Acknowledgements Conflicts of interest}

There are no conflicts of interest.

\section{References}

1 Singnurkar A, Poon R, Metser U. Comparison of 18F-FDG-PET/CT and 18F-FDG-PET/MR imaging in oncology: a systematic review. Ann Nucl Med 2017; 31:366-378.

2 Rosenkrantz AB, Friedman K, Chandarana H, Melsaether A, Moy L, Ding $\mathrm{YS}$, et al. Current status of hybrid PET/MRI in oncologic imaging. Am J Roentgenol 2016; 206:162-172.

3 Schwenzer NF, Schraml C, Muller M, Brendle C, Sauter A, Spengler W, et al. Pulmonary lesion assessment: comparison of whole-body hybrid MR/PET and PET/CT imaging - pilot study. Radiology 2012; 264: 551-558.

4 Reck M, Popat S, Reinmuth N, De Ruysscher D, Kerr KM, Peters S. Metastatic non-small-cell lung cancer (NSCLC): ESMO Clinical Practice Guidelines for diagnosis, treatment and follow-up. Ann Oncol 2014; 25 (Suppl 3):iii27-iii39.

5 Lordick F, Mariette C, Haustermans K, Obermannova R, Arnold D. Oesophageal cancer: ESMO Clinical Practice Guidelines for diagnosis, treatment and follow-up. Ann Oncol 2016; 27(Suppl 5):v50-v57.

6 Bailey DL, Pichler BJ, Guckel B, Barthel H, Beer AJ, Botnar R, et al. Combined PET/MRI: from Status Quo to Status Go. Summary Report of the Fifth International Workshop on PET/MR Imaging; February 15-19, 2016; Tubingen, Germany. Mol Imaging Biol 2016; 18:637-650.

7 Von Schulthess GK, Veit-Haibach P. Workflow considerations in PET/MR imaging. J Nucl Med 2014; 55(Suppl 2):19S-24S.

8 Biederer J, Hintze C, Fabel M. MRI of pulmonary nodules: technique and diagnostic value. Cancer Imaging 2008; 8:125-130.

9 Henzler T, Dietrich O, Krissak R, Wichmann T, Lanz T, Reiser MF, et al. Half-Fourier-acquisition single-shot turbo spin-echo (HASTE) MRI of the lung at 3 Tesla using parallel imaging with 32-receiver channel technology. J Magn Reson Imaging 2009; 30:541-546.

10 Kumar S, Liney G, Rai R, Holloway L, Moses D, Vinod SK. Magnetic resonance imaging in lung: a review of its potential for radiotherapy. $\mathrm{Br} J$ Radio/ 2016; 89:20150431.

11 Biederer J. General requirements of MRI of the lung and suggested standard protocol. In: Kauczor HU, ed. MRI of the lung medical radiology. Berlin, Heidelberg: Springer; 2009.

12 Potchen EJ, Siebert JE, Haacke EM, Gottschalk A. Signal-to-noise, contrast-tonoise, and resolution. In: Patterson AS, ed. Magnetic resonance angiography: concepts \& applications. St. Louis, Missouri, USA: Mosby; 1993.

13 Holland AE, Goldfarb JW, Edelman RR. Diaphragmatic and cardiac motion during suspended breathing: preliminary experience and implications for breath-hold MR imaging. Radiology 1998; 209:483-489.

14 Biederer J, Beer M, Hirsch W, Wild J, Fabel M, Puderbach M, van Beek EJ. MRI of the lung (2/3). Why ... when ... how? Insights Imaging 2012; 3:355-371.
15 Van Rossum PS, van Lier AL, Lips IM, Meijer GJ, Reerink O, van Vulpen $\mathrm{M}$, et al. Imaging of oesophageal cancer with FDG-PET/CT and MRI. Clin Radiol 2015; 70:81-95

16 Lever FM, Lips IM, Crijns SP, Reerink O, van Lier AL, Moerland MA, et al. Quantification of esophageal tumor motion on cine-magnetic resonance imaging. Int J Radiat Oncol Biol Phys 2014; 88:419-424.

17 Dutta J, Huang C, Li Q, El Fakhri G. Pulmonary imaging using respiratory motion compensated simultaneous PET/MR. Med Phys 2015; 42:4227-4240.

18 Biederer J, Mirsadraee S, Beer M, Molinari F, Hintze C, Bauman G, et al. MRI of the lung (3/3)-current applications and future perspectives. Insights Imaging 2012; 3: 373-386.

19 Biederer J, Ohno Y, Hatabu H, Schiebler ML, van Beek EJR, Vogel-Claussen. J, Kauczor. HU. Screening for lung cancer: Does MRI have a role? Eur J Radiol 2017; 86:353-360.

20 Ishii S, Hara T, Nanbu T, Suenaga H, Sugawara S, Kuroiwa D, et al. Optimized workflow and imaging protocols for whole-body oncologic PET/MRI. Jpn J Radiol 2016; 34:754-762.

21 Schaarschmidt BM, Grueneisen J, Heusch P, Gomez B, Beiderwellen K, Ruhlmann V, et al. Oncological whole-body staging in integrated (18)F-FDG PET/MR: value of different MR sequences for simultaneous PET and MR reading. Eur J Radiol 2015; 84:1285-1292.

22 Lee G, I H, Kim SJ, Jeong YJ, Kim IJ, Pak K, et al. Clinical implication of $\mathrm{PET} / \mathrm{MR}$ imaging in preoperative esophageal cancer staging: comparison with PET/CT, endoscopic ultrasonography, and CT. J Nucl Med 2014; 55:1242-1247.

23 Schaarschmidt BM, Grueneisen J, Metzenmacher M, Gomez B, Gauler T, Roesel $\mathrm{C}$, et al. Thoracic staging with F-FDG PET/MR in non-small cell lung cancer - does it change therapeutic decisions in comparison to F-FDG PET/CT? Eur Radiol 2017; 27:681-688.

24 Rauscher I, Eiber M, Furst S, Souvatzoglou M, Nekolla SG, Ziegler SI, et al. $\mathrm{PET} / \mathrm{MR}$ imaging in the detection and characterization of pulmonary lesions: technical and diagnostic evaluation in comparison to PET/CT. J Nucl Med 2014; 55:724-729.

25 Huellner MW, de Galiza Barbosa F, Husmann L, Pietsch CM, Mader CE, Burger IA, et al. TNM staging of non-small cell lung cancer: comparison of PET/MR and PET/CT. J Nucl Med 2016; 57:21-26.

26 Heusch P, Buchbender C, Kohler J, Nensa F, Gauler T, Gomez B, et al. Thoracic staging in lung cancer: prospective comparison of 18F-FDG PET/ MR imaging and 18F-FDG PET/CT. J Nucl Med 2014; 55:373-378.

27 Spick C, Herrmann K, Czernin J. 18F-FDG PET/CT and PET/MRI perform equally well in cancer: evidence from studies on more than 2,300 patients. $J$ Nucl Med 2016; 57:420-430.

28 Qu J, Zhang H, Wang Z, Zhang F, Liu H, Ding Z, et al. Comparison between free-breathing radial VIBE on 3-T MRI and endoscopic ultrasound for preoperative T staging of resectable oesophageal cancer, with histopathological correlation. Eur Radio/ 2018; 28:780-787.

29 Shin HJ, Kim MJ, Lee MJ, Kim HG. Comparison of image quality between conventional VIBE and radial VIBE in free-breathing paediatric abdominal MRI. Clin Radiol 2016; 71:1044-1049.

30 Bamrungchart S, Tantaway EM, Midia EC, Hernandes MA, Srirattanapong S, Dale BM, Semelka RC. Free breathing three-dimensional gradient echo-sequence with radial data sampling (radial 3D-GRE) examination of the pancreas: comparison with standard 3D-GRE volumetric interpolated breathhold examination (VIBE). J Magn Reson Imaging 2013; 38:1572-1577.

31 Dewes P, Frellesen C, Al-Butmeh F, Albrecht MH, Scholtz JE, Metzger SC, et al. Comparative evaluation of non-contrast CAIPIRINHA-VIBE 3T-MRI and multidetector CT for detection of pulmonary nodules: in vivo evaluation of diagnostic accuracy and image quality. Eur J Radiol 2016; 85:193-198.

32 Kuperman V. Magnetic resonance imaging: physical principles and applications. University of Chicago, Illinois: Academic Press; 2000.

33 Van Rossum PS, van Lier AL, van Vulpen M, Reerink O, Lagendijk JJ, $\mathrm{Lin} \mathrm{SH}$, et al. Diffusion-weighted magnetic resonance imaging for the prediction of pathologic response to neoadjuvant chemoradiotherapy in esophageal cancer. Radiother Oncol 2015; 115:163-170.

34 Alper F, Turkyilmaz A, Kurtcan S, Aydin Y, Onbas O, Acemoglu H, Eroglu A. Effectiveness of the STIR turbo spin-echo sequence MR imaging in evaluation of lymphadenopathy in esophageal cancer. Eur J Radio/ 2011; 80:625-628.

35 Schaarschmidt BM, Buchbender C, Nensa F, Grueneisen J, Gomez B, Köhler J, et al. Correlation of the apparent diffusion coefficient (ADC) with the standardized uptake value (SUV) in lymph node metastases of nonsmall cell lung cancer (NSCLC) patients using hybrid 18F-FDG PET/MRI. PLoS One 2015; 10:e0116277. 
36 Heusch P, Buchbender C, Kohler J, Nensa F, Beiderwellen K, Kühl H, et al. Correlation of the apparent diffusion coefficient (ADC) with the standardized uptake value (SUV) in hybrid 18F-FDG PET/MRI in non-small cell lung cancer (NSCLC) lesions: initial results. RoFo 2013; 185:1056-1062.

37 Taouli B, Beer AJ, Chenevert T, Collins D, Lehman C, Matos C, et al. Diffusion-weighted imaging outside the brain: Consensus statement from an ISMRM-sponsored workshop. J Magn Reson Imaging 2016; 44:521-540.

38 Karki K, Saraiya S, Hugo GD, Mukhopadhyay N, Jan N, Schuster J, et al. Variabilities of magnetic resonance imaging-, computed tomography-, and positron emission tomography-computed tomography-based tumor and lymph node delineations for lung cancer radiation therapy planning. Int $J$ Radiat Oncol Biol Phys 2017; 99:80-89.

39 Paulus DH, Oehmigen M, Gruneisen J, Umutlu L, Quick HH. Whole-body hybrid imaging concept for the integration of $\mathrm{PET} / \mathrm{MR}$ into radiation therapy treatment planning. Phys Med Biol 2016; 61:3504-3520.

40 Thorwarth D, Leibfarth S, Mönnich D. Potential role of PET/MRI in radiotherapy treatment planning. Clin Trans/ Imaging 2013; 1:45-51.

41 Bailey DL, Pichler BJ, Guckel B, Antoch G, Barthel H, Bhujwalla ZM, et al. Combined PET/MRI: Global Warming-Summary Report of the 6th International Workshop on PET/MRI, March 27-29, 2017, Tubingen, Germany. Mol Imaging Biol 2018; 20:4-20. 